mining the coefficients of expansion of liquids with known floats, or coefficients of expansion of floats with known liquids. Therefore, any substance which may be made into a float may profitably be studied as to its coefficient of expansion in this way; and such studies will be made in the near future in the Wolcott Gibbs Memorial Laboratory.

The authors take pleasure in expressing their gratitude to the Carnegie Institution of Washington for generous help in the prosecution of this work.

\title{
Summary.
}

In this preliminary paper, experiments are described showing how the phenomenon of floating equilibrium may be used to standardize thermometers. The method consists in determining the temperatures, at which different analyzed solutions of a given substance attain exactly the density of a given float. When the almost linear curve, showing the relation of this floating equilibrium-temperature to percentage composition, has once been established, it will serve for the calibration of any thermometer at any time.

The use of the method as a convenient means of determining the coefficients of expansion of liquids with known floats, or coefficients of expansion of floats with known liquids is also indicated.

Further work upon the subject is now being prosecuted in the Wolcott Gibbs Memorial Laboratory of Harvard University.

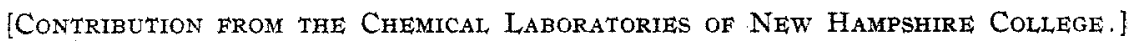

\section{THE DIMETHYL PHOSPHATES OF THE RARE EARTHS.}

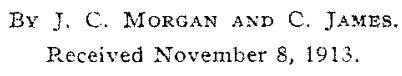

The study of methods for the separation of the rare earths, and of new compounds of the rare earths that seem likely to be of use for such purposes, has, for a long time, occupied the attention of those interested in this branch of chemistry.

The rare earth salts of dimethyl phosphoric acid seemed, on preliminary investigation, to be possessed of properties which would make dimethyl phosphoric acid an extremely valuable reagent for the separation of some of the rare earths. Therefore, a study of the properties of these compounds was commenced.

This investigation involved the preparation of dimethyl phosphoric acid; the preparation of the rare earth compounds from this; the determination of the composition and solubility of these compounds; and finally several fractionations of rare earth mixtures.

Preparation of Dimethyl Phosphoric Acid.-This acid was prepared, according to the method of Hugo Schiff, ${ }^{1}$ by allowing methyl alcohol to

i Chem. Centralblatt, $1857,761-763,864$. 
drop slowly into phosphorus oxychloride. About $200 \mathrm{cc}$. of phosphorus oxychloride were placed in a liter flask and $250 \mathrm{cc}$. of methyl alcohol were added, drop by drop, through a separatory funnel fitted to the flask. During the reaction, the flask was shaken in a stream of cold water and the temperature maintained between $25^{\circ}$ and $30^{\circ}$. The large quantities of hydrochloric acid gas and methyl chloride which were evolved, passed off through a side tube. At the completion of the reaction, which required from an hour to an hour and a half, there remained a colorless, somewhat syrupy liquid, consisting of both dimethyl phosphoric and phosphoric acids with some hydrochloric acid and methyl chloride. This was removed to an evaporating dish and heated upon the steam bath until the hydrochloric acid and methyl chloride were expelled. The syrupy acids were then diluted and neutralized with barium carbonate. The phosphoric acid was removed as barium phosphate. The precipitate was filtered off, leaving a clear filtrate consisting of a solution of barium dimethyl phosphate, together with traces of barium monomethyl phosphate. This solution was treated with just sufficient sulfuric acid to precipitate all the barium as the sulfate, which after digestion at $95^{\circ}$ was removed by filtration, leaving a clear solution of dimethyl phosphoric acid.

Yttrium Dimethyl Phosphate.-This salt was obtained by dissolving yttrium hydroxide in dilute dimethyl phosphoric acid. After stirring for some time, the liquid became clear. The solution was then filtered and placed upon a water bath, the temperature of the latter being kept at about $90^{\circ}$. This treatment caused a quantity of crystalline yttrium dimethyl phosphate to form. The precipitate was filtered off and washed with boiling water. The compound was next dissolved in as small an amount of water as possible, and permitted to crystallize by spontaneous evaporation. Groups of white needle-like crystals, radiating from a common center, were formed. These were removed, dried between filter papers, and then placed in an oven at $100^{\circ}$ for four hours. The results of the analysis seemed to show that a very small amount of water was retained. Further drying at the same temperature failed to make any difference.

Yttrium dimethyl phosphate dissolves in water at $25^{\circ}$ to the extent of 2.80 parts of the anhydrous salt to roo parts of water. At $95^{\circ}$ only about 0.55 parts dissolve in 100 parts of water.

Calculated: $\mathrm{Y}_{2} \mathrm{O}_{8}, 24.34$; found: 24.06 .

Lanthanum Dimethyl Phosphate.-This substance was prepared by dissolving the oxide in dilute dimethyl phosphoric acid. The solution, after filtration, was concentrated upon a water bath until a skin began to form upon the surface, after which it was permitted to crystallize by spontaneous evaporation. The resulting crystals were dissolved in water and recrystallized once again by spontaneous evaporation. These crystals 
were separated from excess of mother liquor by pressing between filter papers and finally dried in air.

Lanthanum dimethyl phosphate forms white hexagonal crystals. One hundred parts of water dissolve 103.7 parts of the anhydrous salt at $25^{\circ}$. The solubility at $100^{\circ}$ did not vary like that of the other rare earths. The solubility at $95^{\circ}$ appeared to be somewhat difficult to determine, owing to the fact that the solution had a tendency to become colloidal.

In order to determine the phosphorus, a weighed sample of lanthanum dimethyl phosphate was first fused with sodium peroxide to oxidize the methyl groups and get the phosphorus present as phosphate. The usual method was then followed.

Calculated: $\mathrm{La}_{2} \mathrm{O}_{3}, 29.63 ; \mathrm{P}_{2} \mathrm{O}_{5}, 38.75$ found: $\mathrm{La}_{2} \mathrm{O}_{3}, 29.45 ; \mathrm{P}_{2} \mathrm{O}_{5}, 38.63$.

From the above analysis, it was considered that the compound contained four molecules of water of crystallization, as shown by the formula: $\mathrm{La}_{2}\left[\left(\mathrm{CH}_{3}\right)_{2} \mathrm{PO}_{4}\right]_{6.4} \dot{\mathrm{H}}_{2} \mathrm{O}$ :

Cerous Dimethyl Phosphate.-The cerous compound was obtained by treating cerous carbonate with a dilute solution of the acid. The clear solution was evaporated on the water bath until a skin began to form upon the surface, as was observed in the case of the preceding substance. It was further concentrated by spontaneous evaporation. The crystals obtained in this manner were again crystallized from water.

Cerous dimethyl phosphate is a white crystalline solid belonging to the hexagonal system. It is very soluble in cold water, but less soluble in hot. One hundred parts of water dissolve 79.6 parts of the anhydrous salt at $25^{\circ}$, and about 65 parts at $95^{\circ}$. Upon analysis $33.08 \% \mathrm{CeO}_{2}$ was found. This still remained after drying for some time at $100^{\circ}$, and corresponded to the formula: $\mathrm{Ce}_{2}\left[\left(\mathrm{CH}_{3}\right)_{2} \mathrm{PO}_{4}\right]_{6} \cdot \mathrm{H}_{2} \mathrm{O}$.

Praseodymium Dimethyl Phosphate.--A solution of this dimethyl phosphate was prepared by dissolving praseodymium oxide in the acid. The liquid was concentrated on the water bath until small crystals began to form, after which it was set aside to evaporate at ordinary temperatures. The compound, purified by recrystallization, formed green, hexagonal crystals. 64.I parts are dissolved by roo parts of water at $25^{\circ}$.

Neodymium Dimethyl Phosphate was prepared in a similar manner to the praseodymitm compound. It forms pale lilac-colored hexagonal plates. At $25^{\circ}, 5^{6.1}$ parts dissolve in roo parts of water, while at $95^{\circ}$, only about 22.3 parts are dissolved by 100 parts of water. An analysis showed the presence of $32.27 \%$ of $\mathrm{Nd}_{2} \mathrm{O}_{3}$, thus pointing to the formula: $\mathrm{Nd}_{2}\left[\left(\mathrm{CH}_{3}\right)_{2} \mathrm{PO}_{4}\right]_{\text {. }}$.

Samarium Dimethyl Phosphate.--A small quantity of the acid was neutralized with samarium oxide. The solution thus obtained was concentrated on the water bath until nearly solid, owing to the formation of 
the dimethyl phosphate crystals. It was filtered while still hot. The salt was dissolved in water and crystallized by evaporation at ordinary room temperature.

Samarium dimethyl phosphate forms cream-colored hexagonal prisms. One hundred parts of water dissolve 35.2 parts of the salt at $25^{\circ}$ and about Io. 8 parts at $95^{\circ}$. $33.11 \% \mathrm{Sa}_{2} \mathrm{O}_{3}$ was found to be present, indicating no water of crystallization like most of the rare earth dimethyl phosphates.

Gadolinium Dimethyl Phosphate was separated from its solution by carefully heating.

It forms white, needle-like crystals very similar to the yttrium compound. One hundred parts of water dissolve 23.0 parts of the salt at $25^{\circ}$, while only 6.7 parts are dissolved by the same quantity at about $95^{\circ}$. The compound contained $34.00 \% \mathrm{Gd}_{2} \mathrm{O}_{3}$.

Erbium Dimethyl Phosphate was prepared in a similar way to the ytterbium salt described below.

It forms very pale-colored needles, 1.78 parts dissolving in roo parts of water at $25^{\circ}$.

Ytterbium Dimethyl Phosphate.-The ytterbium oxide was dissolved in a slight excess of hydrochloric acid, and the solution diluted considerably. A sufficient quantity of dimethyl phosphoric acid to react with the ytterbium present was neutralized with sodium carbonate, and then made slightly acid with a few drops of dimethyl phosphoric acid. This solution was diluted and slowly added to the ytterbium chloride with careful stirring. A precipitate of ytterbium dimethyl phosphate formed, which slightly increased in amount on heating the solution to $100^{\circ}$. The precipitate was filtered off, dissolved in cold water, and again precipitated by heating. The white needle-like crystals dissolve to the extent of 1.2 parts per roo parts of water at $25^{\circ}$. Only 0.25 is dissolved by roo parts of water at about $95^{\circ}$. An analysis showed $35.73 \% \quad \mathrm{Yb}_{2} \mathrm{O}_{3}$, which corresponds to the amount contained in the formula of the anhydrous substance.

TABLE OF SOlubilities of Dimethyl Phosphates.

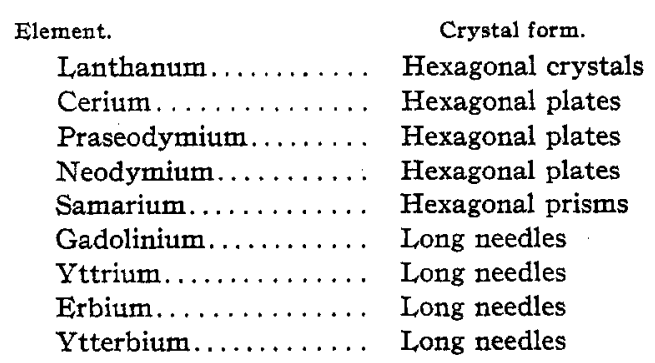

Parts of salt per 100
parts of water at $25^{\circ}$.
I03.7
79.6
64.1
56.1
35.2
23.0
2.8
1.78
1.2

Fractionation of Material.-Since, as already stated, the study of new compounds of the rare earths is carried on mainly to obtain compounds adaptable to a more complete and more rapid separation of these elements, 
no study would be complete without determining the behavior of mixtures of the dimethyl phosphates. Several fractionations were conducted, and the method employed was that of precipitation by heat. In the case of the less soluble compounds, a dilute solution was prepared. The beaker containing this solution was then placed in a water bath and the temperature of the bath gradually raised, the solution being constantly stirred. As soon as a fair amount of precipitate had formed, the liquid was filtered off and the precipitate retained as Fraction $I$. The filtrate was again heated until another lot had separated, and again filtered. 'The dimethyl phosphates collected from this second heating were put aside as Fraction II. In this manner fractions were taken up to and inclusive of $95^{\circ}$. Additional fractions were obtained by fractionally evaporating the mother liquor.

These fractions were further fractionated by dissolving the least soluble fraction in water, and heating in the water bath to the temperature at which Fraction $I$ was removed. The precipitate which had formed at this point was removed by filtration and retained as Fraction I-2. The filtrate was used to dissolve Fraction II, and the solution heated as before to the temperature of removal of Fraction II. The precipitate was taken as Fraction II-2, and the filtrate used to dissolve Fraction III. By proceeding in this manner any degree of fractionation, which was desired, could be obtained.

During, and at the completion of the fractionation, various fractions were examined with regard to color of oxide, nitric acid solution, and spectrum.

Gadolinim Material.-A material, containing gadolinium with only sufficient traces of terbium to color the oxide orange-brown, was first submitted to fractionation with dimethyl phosphoric acid.

The oxide of this crude gadolinium was dissolved by stirring with the acid until all had dissolved. The liquid was then treated as described above.

The fractions were taken as follows: Fractions I, I-2, I-3 at 65 ; Fractions II, II-2, II-3 at $90^{\circ}$; Fractions III, III-2, III-3, after evaporating the mother liquor from Fractions II to one-half volume; and Fractions IV, IV-2, IV-3, upon complete evaporation.

A fair idea can be formed, with regard to the rapidity of the method, by observing the change in color of the oxides. The oxide from Fraction II was of a dark brown color, that of Fraction IV light brown, and that of Fraction IV-3 pale cream.

This showed a rapid concentration of the gadolinium in the most soluble fractions, while the impurity-terbium-collected in the least soluble.

It is interesting to note that this fractionation required only about forty-eight hours to give a comparatively pure gadolinium. This shows a great increase in the rapidity of the separation over all previous methods. 
Fractionation of Yttrium Material.-The next oxides consisted of a mixture of those of yttrium, holmium and dysprosium with traces of erbium, samarium, gadolinium, terbium, neodymium and praseodymium. They were dissolved in hydrochloric acid, the solution diluted and boiled with an excess of sodium hydroxide. The precipitated hydroxides were filtered off and washed with hot water until free from chlorides and dissolved by stirring with the dilute dimethyl phosphoric acid. The resulting solution was then submitted to the usual fractionation.

Fraction I was removed at $38^{\circ}$, Fraction II at $48^{\circ}$, Fraction III at $65^{\circ}$, Fraction IV at $96^{\circ}$, while Fractions V, VI, VII and VIII were taken by fractionally evaporating the mother liquor from Fraction IV.

Fraction I.-The oxide was yellowish, and the absorption spectrum showed a rapid concentration of erbium. Holmium and dysprosium were also present.

Fraction II.-This portion gave a yellowish colored oxide. The spectroscope showed holmium and dysprosium with very small quantities of erbium.

Fraction VI.-The oxide was colored an orange-brown. An intense absorption proved the presence of very much dysprosium, less holmium, and the merest possible trace of neodymium.

Fraction VII.-Reddish brown oxide. The absorption spectrum was very weak, showing the presence of a small amount of neodymium and traces of samarium and dysprosium. The green band of holmium could barely be detected.

Fraction VIII.-This gave an orange-brown oxide. The spectrum showed intense absorption bands, indicating the presence of a large quantity of neodymium, a very little praseodymium, and only a trace of samarium.

Fractionation of a mixture of earths, from monazite, giving more soluble double sulfates. The earths present consisted largely of gadolinium and dysprosium with small amounts of terbium, holmium and neodymium.

The oxides were warmed with dimethyl phosphoric acid until entirely converted into dimethyl phosphates, after which the thick mass was stirred with water until dissolved.

The fractions were collected as follows: Fractions I to III up to $95^{\circ}$; and Fractions IV and V by evaporation of the mother liquor from Fraction III.

Fraction I gave a brownish yellow oxide, which, when dissolved in nitric acid, gave a yellowish green solution. With the aid of the spectroscope, it was found that large amounts of dysprosium were present, accompanied by a little holmium.

Fraction II.-The oxide was chocolate-brown. Its nitrate solution was faintly green and showed weak absorption bands of dysprosium and terbium. 
Fraction V.-Oxide red-brown. Absorption spectrum indicated very small quantities of neodymium and dysprosium.

It will be observed from the above fractionations that the rate of separation of the rare earths is vastly greater than practically all the methods given up to the present time. Lanthanum, cerium, praseodymium, neodymium are left at once in the mother liquor. Samarium, europium, and gadolinium are much less soluble than those previously mentioned, while they are more soluble than terbium, dysprosium and holmium. Erbium, thulium, yttrium, ytterbium, etc., collect in the least soluble portions.

Since the solubilities of these compounds are the reverse of the usual type, they may be used for the rapid purification of many of the rare earths. For instance, we can easily remove traces of neodymium from samarium by this means, as the samarium dimethyl phosphate separates before the neodymium compound.

It is necessary to state that there is some inconvenience when working with the salts of dimethyl phosphoric acid, since they undergo a very gradual decomposition. A gelatinous precipitate is formed, very slowly, in the case of the rare earths, which filters with difficulty.

DURHaM, N. H.

\section{THE UTILIZATION OF DIFFUSION PROCESSES IN THE PREPARATION OF PURE SUBSTANCES.}

\footnotetext{
BY JOHN JOHNSTON.

Received October 27, 1913.
}

The possibilities of utilizing diffusion processes in preparing certain substances in the form of relatively large crystals appear to be much less widely known than they deserve to be, in view of the simplicity of the procedure and the purity of the resultant product. This type of method is quite generally applicable; its use is especially advantageous in the formation, by precipitation, of sparingly soluble substances. Such substances, as ordinarily prepared, tend to separate out in very fine powder which, by reason of its large extent of surface, often contains much absorbed material, in which case they are extremely difficult to purify satisfactorily. By allowing the solutions to mix by the operation of diffusion we may ensure very slow precipitation, and in this way obtain large, well-developed crystals, which can easily be washed free from any adherent foreign material.

The size of grain of a precipitate $A D\left(e, g\right.$., $\left.\mathrm{Ca}\left(\mathrm{OH}_{2}\right)\right)$, formed by the interaction of solutions $A B$ and $C D$ (e.g., $\mathrm{CaCl}_{2}$ and $\mathrm{NaOH}$ ), depends upon the virtual concentration in the mixed solution of the ions $A^{+}$and $D^{\prime}$, relative to their concentrations in the solution which, under the conditions of experiment, is saturated with respect to $A D$. This was demonstrated directly by von Veimarn for precipitates of barium sulfate; ${ }^{k}$

${ }^{1}$ P. P. von Veimarn, Z. Chem. Ind. Kolloide, 2, 3. 